JOURNAL OF SYNCHROTRON RADIATION

ISSN 1600-5775

Received 27 March 2020

Accepted 17 July 2020

Edited by V. Favre-Nicolin, CEA and Université Joseph Fourier, France

₹ These authors contributed equally to this work.

Keywords: nanoprobe X-ray exposure; individual core-shell nanowires; morphological and optical changes; ozone-induced oxidation; air and He atmosphere.

Supporting information: this article has supporting information at journals.iucr.org/s

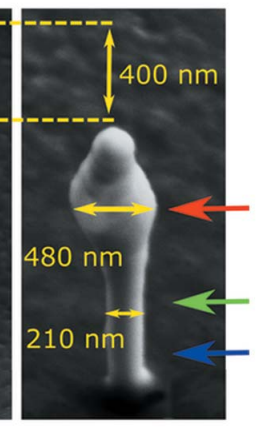

OPEN $\odot$ ACCESS

\section{Beam damage of single semiconductor nanowires during $X$-ray nanobeam diffraction experiments}

\author{
Ali Al Hassan, ${ }^{\mathrm{a} *} \ddagger$ Jonas Lähnemann, ${ }^{\mathrm{b}} ¥$ Arman Davtyan, ${ }^{a}$ Mahmoud Al-Humaidi, \\ Jesús Herranz, ${ }^{\mathrm{b}}$ Danial Bahrami, ${ }^{\mathrm{a}}$ Taseer Anjum, ${ }^{\mathrm{a}}$ Florian Bertram, ${ }^{\mathrm{c}}$ Arka Bikash \\ Dey, ${ }^{\mathrm{C}}$ Lutz Geelhaar ${ }^{\mathrm{b}}$ and Ullrich Pietsch ${ }^{\mathrm{a}}$
}

\footnotetext{
${ }^{a}$ Naturwissenschaftlich-Technische Fakultät der Universität Siegen, Siegen 57068, Germany,

${ }^{\text {b}}$ Paul Drude Institut für Festkorperelektronik, Leibniz Institut im Forschungsverbund Berlin e.V., Hausvogteiplatz 5-7, Berlin 10117, Germany, and ' DESY Photon Science, Notkestrasse 85, Hamburg 22607, Germany.

*Correspondence e-mail: ali.alhassan@uni-siegen.de
}

Nanoprobe X-ray diffraction (nXRD) using focused synchrotron radiation is a powerful technique to study the structural properties of individual semiconductor nanowires. However, when performing the experiment under ambient conditions, the required high X-ray dose and prolonged exposure times can lead to radiation damage. To unveil the origin of radiation damage, a comparison is made of nXRD experiments carried out on individual semiconductor nanowires in their as-grown geometry both under ambient conditions and under $\mathrm{He}$ atmosphere at the microfocus station of the P08 beamline at the thirdgeneration source PETRA III. Using an incident X-ray beam energy of $9 \mathrm{keV}$ and photon flux of $10^{10} \mathrm{~s}^{-1}$, the axial lattice parameter and tilt of individual $\mathrm{GaAs} / \mathrm{In}_{0.2} \mathrm{Ga}_{0.8} \mathrm{As} / \mathrm{GaAs}$ core-shell nanowires were monitored by continuously recording reciprocal-space maps of the 111 Bragg reflection at a fixed spatial position over several hours. In addition, the emission properties of the (In,Ga)As quantum well, the atomic composition of the exposed nanowires and the nanowire morphology were studied by cathodoluminescence spectroscopy, energy-dispersive X-ray spectroscopy and scanning electron microscopy, respectively, both prior to and after nXRD exposure. Nanowires exposed under ambient conditions show severe optical and morphological damage, which was reduced for nanowires exposed under $\mathrm{He}$ atmosphere. The observed damage can be largely attributed to an oxidation process from X-ray-induced ozone reactions in air. Due to the lower heat-transfer coefficient compared with GaAs, this oxide shell limits the heat transfer through the nanowire side facets, which is considered as the main channel of heat dissipation for nanowires in the as-grown geometry.

\section{Introduction}

The development of X-ray optics and third-generation synchrotron radiation sources with high-brightness, nanofocused X-ray beams (Martínez-Criado et al., 2016; Leake et al., 2019) facilitates probing the structural parameters, spatial alloy distribution, crystal phases and strain distribution of single nanowires (NWs) by means of X-ray-based methods. These methods include nano-X-ray fluorescence (Al Hassan et al., 2018a), coherent Bragg ptychography (Hill et al., 2018) as well as coherent and non-coherent nanoprobe X-ray diffraction (nXRD) (Biermanns et al., 2013; Stankevič et al., 2015). For example, Al Hassan et al. (2018b) demonstrated that the thicknesses of the core and shells within individual core-shell NWs, as well as the strain distribution, can be accessed in the as-grown geometry by recording reciprocal-space maps (RSMs) of in-plane Bragg reflections. However, during 
the acquisition of RSMs around Bragg reflections, the beam is fixed to a specific position on the NW for an extended time.

The continuous exposure during such experiments will inevitably increase the risk of radiation damage due to an accumulating X-ray dose at the illuminated NW section. For instance, Shi et al. (2012) have demonstrated radiationinduced bending of Si-on-insulator NWs by means of coherent diffraction imaging, i.e. the authors have observed a splitting of the Bragg reflections, which continuously evolves with increasing X-ray dose. Working on similar systems, Mastropietro et al. (2013) quantitatively demonstrated elastic strain relaxation in single Si-on-insulator lines under the influence of prolonged X-ray exposure. This was done by monitoring the evolution of the $11 \overline{3}$ Bragg peak while illuminating the same position for different time intervals. The structural damage induced by the absorbed X-ray dose was shown to occur only when an oxide layer is present under the Si thin film. Whereas Polvino et al. (2008) reported that the exposure induced permanent structural damage to the crystal structure, Mastropietro et al. (2013) have shown that the intense radiation only damages the $\mathrm{Si} / \mathrm{Si}$-on-insulator interface but not the crystalline Si structure. Aside from $\mathrm{SiC}$ and $\mathrm{GaN}$ which potentially offer radiation-hard alternatives to silicon devices (Sellin \& Vaitkus, 2006), bulk or layered GaAs is known to be a very radiation-hard material suitable for X-ray detectors (Claeys \& Simoen, 2002; Lioliou \& Barnett, 2016; Smolyanskiy et al., 2018). Here, we show that GaAs/ (In,Ga)As/GaAs core-shell NWs may also be affected by $\mathrm{X}$-ray-induced radiation damage. We will illustrate in detail the impact of the exposure to high X-ray doses on the structure, morphology and optical emission of individual NWs.

In this study, two separate experiments have been carried out using the same beam conditions, i.e. photon flux, energy and beam size, where individual NWs are continuously exposed at a fixed position along their growth axes. The first experiment is performed under air atmosphere, whereas the second is done under $\mathrm{He}$ atmosphere. The structural changes, e.g. tilting and axial lattice variation, were monitored by continuously recording RSMs of the 111 Bragg reflection as a function of exposure time and absorbed X-ray dose. Scanning electron microscopy (SEM) imaging is used before and after $\mathrm{X}$-ray exposure to observe morphological changes, energydispersive X-ray spectroscopy (EDX) to track compositional changes, and hyperspectral cathodoluminescence (CL) mapping to assess the impact on the optical emission. To facilitate the latter, a NW sample with a core-shell quantum well $(\mathrm{QW})$ geometry is used.

\section{Experimental details}

The investigated NWs were grown by molecular beam epitaxy on a patterned $\mathrm{Si}(111)$ substrate using the Ga-assisted vaporliquid-solid mechanism. The as-grown NWs are about $2.5 \mu \mathrm{m}$ in length and $150 \mathrm{~nm}$ in diameter and contain radial heterostructures of $\mathrm{GaAs} /(\mathrm{In}, \mathrm{Ga}) \mathrm{As} / \mathrm{GaAs}$ with $20 \%$ nominal In concentration, $10 \mathrm{~nm}$ thickness of the (In,Ga)As QW shell and
Table 1

The exposure times in hours and the atmospheres surrounding the four NWs discussed in this paper during the measurements.

\begin{tabular}{lllll}
\hline & NW1 & NW2 & NW3 & NW4 \\
\hline $\begin{array}{lllll}\text { Exposure time (h) } \\
\text { Atmosphere }\end{array}$ & 1 & 4 & 1 & 3 \\
\hline
\end{tabular}

$30 \mathrm{~nm}$ thickness of the outer GaAs shell. The investigated NWs were grown along a straight line on the substrate with a spacing of $10 \mu \mathrm{m}$ between two neighboring NWs (Al Hassan et $a l ., 2018 b$ ). This makes it possible to access the same individual NWs both in nXRD and SEM/CL/EDX measurements. More details about the growth process and sample geometry can be found in the work of Küpers et al. (2018, 2019).

The two nXRD experiments performed under air and $\mathrm{He}$ atmospheres were both carried out at beamline P08 of PETRA III (Seeck et al., 2012) using identical conditions. A photon energy of $9 \mathrm{keV}$ was used, and the photon flux integrated over the cross section of the beam was about $10^{10} \mathrm{~s}^{-1}$, while the vertical and horizontal full width at half-maxima of the beam were $0.6 \mu \mathrm{m}$ and $1.8 \mu \mathrm{m}$, respectively. In order to study the impact of $\mathrm{X}$-ray exposure on the structural properties, NWs were measured for several hours under either air or inert gas atmosphere. A total of $12 \mathrm{NWs}$ were systematically exposed for durations from 1 to $4 \mathrm{~h}$ under ambient conditions. Similarly, five individual NWs were exposed for 1 to $3 \mathrm{~h}$ under $\mathrm{He}$ atmosphere (see the supporting information for details on the experimental implementation). For the four NWs discussed in this paper, the exposure times and the measurement atmospheres are listed in Table 1. To trace structural changes during the exposure, RSMs of the 111 Bragg reflection were continuously recorded at the same position along the NW growth axis. The time needed to record each RSM was about $10 \mathrm{~min}$. The methods used to translate from real space to reciprocal space and to construct a 3D RSM of the measured Bragg reflection can be found in the works of Pietsch et al. (2004) and Al Hassan et al. (2018b).

For all investigated NWs, SEM images and CL hyperspectral line scans were recorded before and after X-ray exposure using a Zeiss Ultra55 field-emission scanning electron microscope operated at an acceleration voltage of $5 \mathrm{kV}$ with beam currents of $0.2-0.3 \mathrm{nA}$. For low-temperature CL measurements at $15 \mathrm{~K}$, the scanning electron microscope is fitted with a Gatan MonoCL4 system and a He-cooled sample stage (Lähnemann et al., 2019). To measure the as-grown NWs, the sample is cleaved close to the line of NWs and mounted at an angle of $45^{\circ}$, which is accounted for when plotting the CL. Line scans are recorded by scanning the beam along the axis of the NW and recording a spectrum for $1 \mathrm{~s}$ at every dwell point. The Python package HyperSpy is used to process the CL data (de la Peña et al., 2019). EDX measurements of selected unexposed and exposed NWs were recorded in the same scanning electron microscope using an EDAX silicon drift detector (Apollo XV) at an acceleration voltage of $3 \mathrm{keV}$, probing the $L$ lines of $\mathrm{Ga}$ and As, as well as the $K$ lines of $\mathrm{C}$, $\mathrm{O}$ and $\mathrm{Si}$. 


\section{Experimental results}

Starting with the experiment under ambient conditions (air atmosphere), the impact of X-ray exposure on the NW structure will be exemplified for two representative NWs out of 12 measured NWs. At the beginning of the exposure, the two NWs display well defined hexagonal cross sections and side facets, as seen in Figs. 1(a) and 1(e).

The first NW, referred to as NW1 in Table 1, was exposed for $1 \mathrm{~h}$, which corresponds to an absorbed dose of $2.5 \times 10^{11}$ Gy (see estimate in Part 3 of the supporting information). After exposure, its diameter increased by $50 \mathrm{~nm}$ which, if assumed to be symmetric, is $25 \mathrm{~nm}$ on the wall of each opposing side facet, and its length increased by $200 \mathrm{~nm}$; see Fig. 1(b). This causes the well defined side facets to disappear. As detailed below, we attribute this increase in the diameter and length of the NW mostly to oxidation of the GaAs initiated by the creation of ozone under the high-intensity X-ray beam. Such an oxidation process under strong UV illumination in air has previously been investigated for planar GaAs layers (Flinn \& McIntyre, 1990; Lu et al., 1993; Hollinger et al., 1994).

The morphological changes were accompanied by a local degradation in the optical properties of the NW. This degradation was visualized by spectrally resolved CL line scans of the emission from the (In, Ga)As QW acquired before [Fig. 1(c)] and after [Fig. 1(d)] exposure. Here, the emission energy is plotted against beam position along the NW axis with the emission intensity color-coded on a logarithmic color scale. Before exposure, the NW QW showed a homogeneous distribution of the luminescence along the NW growth axis. The emission is centered at about $1.25 \mathrm{eV}$ and shows only a minor blue shift between the bottom and center of the NW. The tip of the NW does not emit due to a reduced crystal quality and the absence of the QW in this segment that is formed by axial elongation during shell growth, which also leads to a change in faceting observed in Figs. 1(a) and 1(e) (Lähnemann et al., 2019). After exposure, a significant degradation of the $\mathrm{CL}$ emission is visible in the segment between 1.2 and $1.6 \mu \mathrm{m}$ along the NW axis [Fig. 1(d)], which is assumed to be the position of the X-ray nanobeam.

The second NW, referred to as NW2 in Table 1, was exposed for $4 \mathrm{~h}$, corresponding to an absorbed dose of $10 \times 10^{11} \mathrm{~Gy}$. For NW2, more severe morphological changes are observed after exposure, concerning the outer surface, length and diameter of the NW [see Figs. 1(e) and 1(f)]. First, the NW surface lost its well defined facets, which again can be explained by ozone-induced oxidation. Second, the NW section indicated by a red dashed circle in Fig. 1(e) vanishes and beneath it a swelling-like feature becomes visible in Fig. 1(f). As we discuss below, the top section of the NW, which is approximately $500 \mathrm{~nm}$ in length, has melted and formed the swelling that we observe in Fig. 1(f). As a consequence, the NW diameter is increased to approximately $210 \mathrm{~nm}$ at the bottom and up to $480 \mathrm{~nm}$ at the swollen area. The melting of the NW top section after exposure brings us to the conclusion that the NW section named P in Fig. 1(e) was illuminated by the peak of the Gaussian-shaped X-ray beam, whereas the NW section circled in pink and named T was illuminated by its tail. Due to the submicrometre vertical size and Gaussian profile of the beam, the ozone oxidation as well as the density of the growing oxide are following the local X-ray intensity, i.e. it is maximum at the position of the center of the Gaussian $\mathrm{X}$-ray beam under air atmosphere and less in the tails. The morphological damage of NW2 was accompanied by a complete loss of its CL emission along the whole length of the NW (not shown).

To investigate the elemental composition of the shell formed around the NWs, EDX measurements were carried out at the top, middle and bottom sections of the same NWs measured by $\mathrm{nXRD}$ in air. The resulting spectra for NW2 are shown in Fig. $2(a)$ at the positions marked by arrows in Fig. 1(f), where each spectrum was normalized with respect to the Ga peak. Compared with a NW that was not exposed to the X-ray beam, shown as horizontal pink lines in Fig. 2(a), a significant increase of the $\mathrm{O}$ signal is seen along the whole length of the NW. Towards the swollen top, the O signal is further enhanced and also the $\mathrm{C}$ peak is increased. This

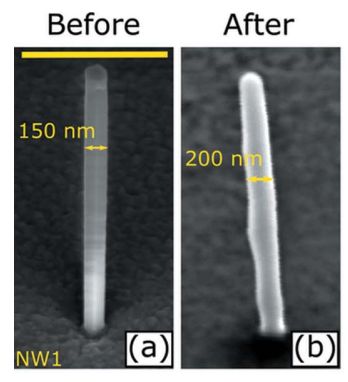

Figure 1

$(a),(b)$ SEM micrographs of NW1 before and after exposure for $1 \mathrm{~h}$, respectively. $(c),(d)$ Normalized low-temperature hyperspectral CL line scans along the NW growth axis before and after exposure (color-coded logarithmic intensity scale), respectively, for NW1. Note that the emission intensity depends sensitively on the positioning of the NW with respect to the focal point of the parabolic mirror so that the absolute intensities of the two line scans cannot be compared directly. $(e),(f)$ SEM micrographs of NW2 before and after exposure for $4 \mathrm{~h}$, respectively. Red and pink dashed circles indicate NW sections illuminated by the peak (P) and tail (T) of the Gaussian beam. Colored arrows indicate the positions at which the EDX measurements shown in Fig. 2 were carried out. The scale bar in $(a)$ corresponds to $1 \mu \mathrm{m}$ and applies to all SEM images. 

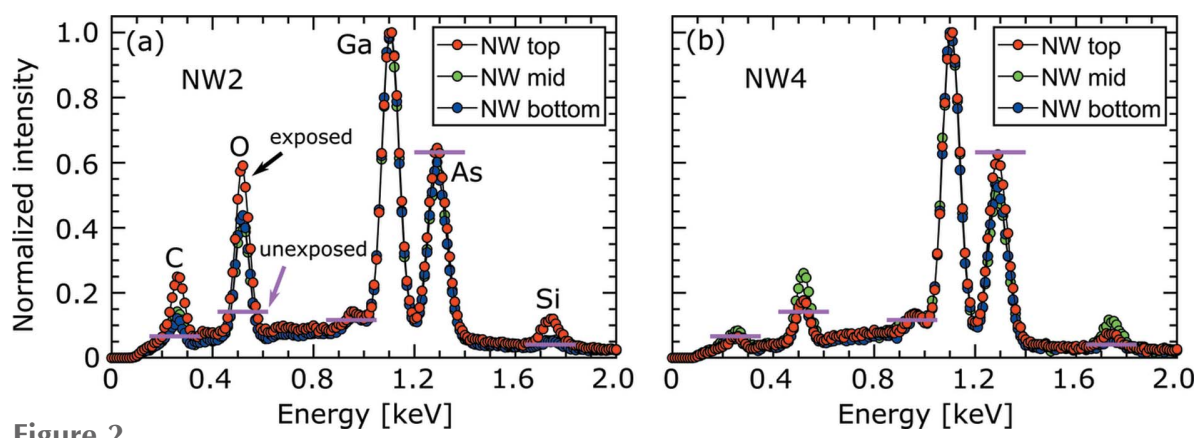

Figure 2

EDX spectra taken at the top, middle and bottom sections of (a) NW2 (air) and (b) NW4 (He). The color of each line profile is correlated with the color of the arrows in Fig. 1(f) for NW2 and in Fig. 3(f) for NW4. The horizontal pink lines correspond to the peak height for each element in a non-exposed NW.

measurement confirms that oxidation of the NW surface takes place, which is likely to be the major driving force for the degradation of the NW morphology and optical properties. However, it is worth noting that the sensitivity of EDX decreases for small characteristic X-ray energies and is thus lowest for the $\mathrm{C}$ peak. The presence of hydrocarbon molecules on the surface is inevitable. These molecules can be cracked by the impinging energetic $\mathrm{X}$-ray beam during exposure, leading to the deposition of amorphous $\mathrm{C}$ on the surface of the NW (Boller et al., 1983). The oxidized NW might lead to an enhanced scattering of electrons into the substrate, which would explain the slight Si signal visible for the exposed NWs.

To reduce the ozone-driven oxidation of the NW crystal structure, we replicate the nXRD experiment using the same beam size, photon flux and energy but under $\mathrm{He}$ atmosphere. Here, He was pumped into a cylinder made from Kapton tape that has been implemented to shield the sample from air (see Fig. S1 in the supporting information). A small hole was drilled at the side wall of the Kapton tape chamber to release the He overpressure. The EDX spectrum for NW4, exposed to the X-ray beam for $3 \mathrm{~h}$ in the $\mathrm{He}$ atmosphere, is shown in Fig. 2(b). A SEM micrograph of this NW, indicating the positions of the EDX measurements, is displayed in Fig. 3(e). As expected, the $\mathrm{O}$ content is significantly reduced for NW4 compared with NW2. Compared with the peak heights for the non-exposed NWs indicated by the horizontal pink lines in
Fig. 2, the $\mathrm{O}$ signal is increased only for the middle section of NW4, which is the beam position during exposure. A small $\mathrm{O}$ signal even under $\mathrm{He}$ atmosphere can be explained by residual $\mathrm{O}$ inside the Kapton chamber.

To further compare the exposure under air and $\mathrm{He}$ atmospheres, Fig. 3 shows SEM micrographs before and after, as well as CL line scans after $\mathrm{X}$-ray exposure for two NWs measured in the He chamber: NW3, exposed for $1 \mathrm{~h}$, and NW4. In contrast to NW1, which was exposed for a similar duration in air, NW3 barely shows any increase in diameter or tilt and its facets still appear well pronounced. In addition, only a minor reduction of the CL intensity is observed after exposure at the mid-section of NW3, whereas the CL emission is locally quenched for NW1. NW4 shows a more significant shell deposition compared with NW3 [Fig. 3(b)] but much less pronounced compared with NW2 or even NW1 [Figs. 1 $(f)$ and 1(b)]. In line with the EDX measurements, NW4 is slightly widened at its mid-section. In contrast to NWs that were exposed for more than $2 \mathrm{~h}$ in air atmosphere, the CL emission of NW4 is not completely quenched, i.e. it is still visible at the bottom [Fig. 3(f)].

\subsection{RSMs of the 111 Bragg reflection}

In the following, we present a detailed analysis of nXRD measurements reflecting the beam-induced damage as a function of exposure time. We continuously recorded RSMs of the 111 Bragg reflection on the individual NWs. Starting with the NWs exposed in air atmosphere, a selection of typical $\left(Q_{Z}^{111}, Q_{Y}^{111}\right)$ and $\left(Q_{Y}^{111}, Q_{X}^{111}\right) 2 \mathrm{D}$ projections of the 3D 111 Bragg reflection of NW2 are presented in Figs. 4(a)-4(e) and Figs. $4(f)-4(j)$, respectively. Here, $Q_{Z}^{111}$ is defined along the scattering direction of the 111 Bragg reflection in reciprocal space and is sensitive to polytypism and variation in the axial $c$ lattice parameter. The reciprocal-space vectors $Q_{X}^{111}$ and $Q_{Y}^{111}$ are defined along the [22 $\overline{4}]$ and [220] directions of the NW,

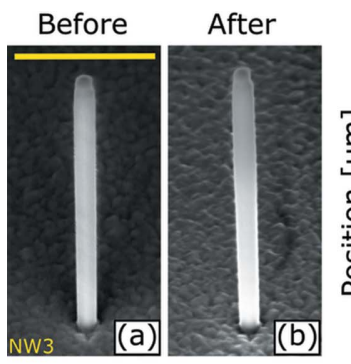

Figure 3

(a), (b) SEM micrographs of NW3 before and after exposure for $1 \mathrm{~h}$, respectively. (c) Normalized low-temperature hyperspectral CL line scan along the NW growth axis after exposure (color-coded on a logarithmic intensity scale) for NW3. $(d)$, (e) SEM micrographs of NW4 before and after exposure for $3 \mathrm{~h}$, respectively. Colored arrows indicate the positions at which EDX measurements were carried out. $(f)$ Normalized low-temperature hyperspectral CL line scan after exposure for NW4. The scale bar in $(a)$ corresponds to $1 \mu \mathrm{m}$ and applies to all SEM images. 


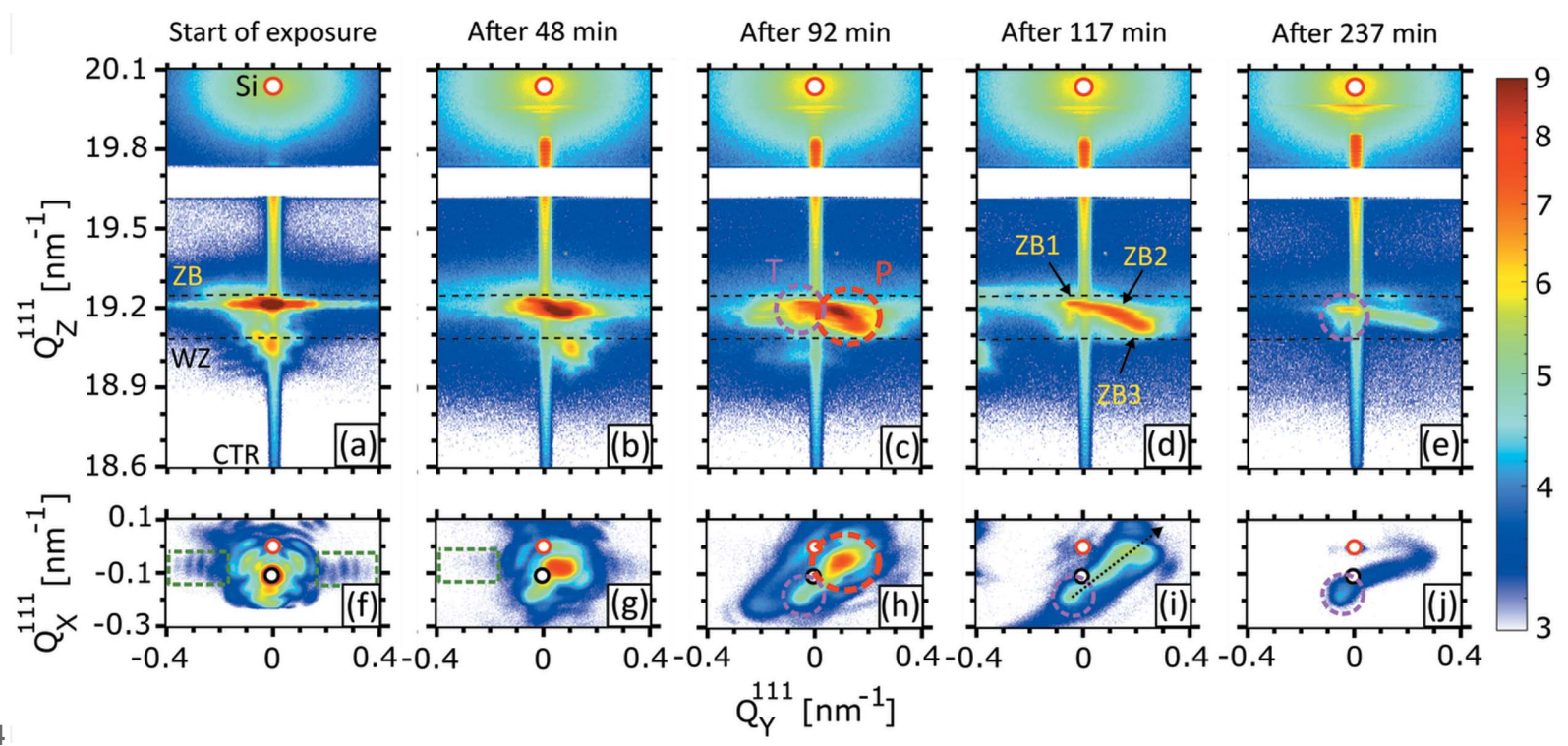

Figure 4

(a)-(e) 2D projections of the 111 Bragg reflection in the $\left(Q_{Z}^{111}, Q_{Y}^{111}\right)$ reciprocal-space plane for NW2 exposed in air. The two dashed curves in $Q_{Z}^{111}$ represent the ZB (top) and WZ (bottom) Debye-Scherrer rings. The red and pink dotted circles named $\mathrm{P}$ and T are explained in Fig. 1(e) and represent sub-Bragg peaks that originate from NW sections illuminated by the peak and tail of the Gaussian beam. The peaks named ZB1-ZB3 are referred to in Fig. $6(d) .(f)-(j) 2$ D projections of the 111 Bragg reflection in the $\left(Q_{Y}^{111}, Q_{X}^{111}\right)$ reciprocal-space plane. The red and black circles represent the positions of the Si CTR and the initial NW tilt, respectively, in $Q_{X}^{111}$ and $Q_{Y}^{111}$. The time at which each RSM acquisition was started is mentioned at the top.

respectively, and are sensitive to both the NW thickness and tilt. The RSMs in Figs. 4(a), 4(f) have been recorded only $3.6 \mathrm{~min}$ after the start of the exposure. The Si 111 Bragg reflection in the upper part of the RSM was considered as a reference to calculate the variation in the axial lattice spacing of the NW and therefore was placed at the unstrained position of $Q_{Z}^{111}=20.038 \mathrm{~nm}^{-1}$. Apart from $\mathrm{Si}$, the pseudomorphic GaAs zincblende $(\mathrm{ZB})\left(Q_{Z}^{111} \simeq 19.22 \mathrm{~nm}^{-1}\right)$ and wurtzite (WZ) $\left(Q_{Z}^{111} \simeq 19.06 \mathrm{~nm}^{-1}\right)$ Bragg reflections are visible. The nominal positions for unstrained GaAs of both polytypes, $Q_{Z}^{111} \simeq 19.25 \mathrm{~nm}^{-1}$ for ZB and $Q_{Z}^{111} \simeq 19.09 \mathrm{~nm}^{-1}$ for WZ, are indicated by dashed Debye-Scherrer rings in all $\left(Q_{Z}^{111}, Q_{Y}^{111}\right)$ RSMs. Both reflections are slightly shifted from the unstrained positions due to the lattice mismatch between GaAs and the (In, Ga)As shell with nominal indium content of $20 \%$. After 48 min of X-ray exposure, the ZB reflection elongates towards smaller $Q_{Z}^{111}$ values and shifts along $Q_{Y}^{111}$, giving evidence for lattice expansion and tilt. The WZ peak also moves towards lower $Q_{Z}^{111}$ values. After $92 \mathrm{~min}$, the main peak splits into two sub-peaks. Considering Fig. 1(e), the sub-peaks circled in pink and red resemble the NW sections assumed to be illuminated by the tail and peak of the Gaussian nanobeam, respectively. At the end of the exposure, the sub-peak originating from section $\mathrm{P}$ vanishes, which is attributed to the melting of this NW section as observed in Fig. 1(f).

In the $\left(Q_{Y}^{111}, Q_{X}^{111}\right)$ RSMs, the small red and black circles correspond to the Si crystal truncation rod (CTR) and the NW tilt at the beginning of exposure, respectively. The thickness oscillations present in Fig. 4(f) and indicated by a green, dashed rectangle correspond to a NW diameter of $154 \pm 5 \mathrm{~nm}$, which is in very good agreement with the SEM observation. From the appearance of thickness oscillations of the ZB reflection, it is evident that the beam is well aligned on the
NW. After 48 min [Fig. 4(g)], the thickness oscillations disappear, which may be explained by the amorphous layer created by the oxidation of GaAs. Starting after $92 \mathrm{~min}$ of exposure, similar to the $\left(Q_{Z}^{111}, Q_{Y}^{111}\right)$ maps, the Bragg peak divides into two sub-peaks. The first sub-peak, denoted by T, remains at the same $Q_{X}^{111}$ and $Q_{Y}^{111}$ positions showing no tilt, whereas the second sub-peak, named $\mathrm{P}$, splits from the first one and moves along the dashed arrow in Fig. 4(i). At the end of the exposure (after $240 \mathrm{~min}$ ), P vanishes. The RSMs of NW1 are given in the supporting information (Fig. S2) and show a behavior similar to that of the RSMs of NW2 recorded during the first hour of exposure.

Similarly, several individual NWs have been exposed under $\mathrm{He}$ atmosphere at a fixed position along their growth axes for different time intervals while the RSMs of the $111 \mathrm{Bragg}$ reflection were continuously recorded. Fig. 5 shows the $\left(Q_{Z}^{111}, Q_{Y}^{111}\right)$ and $\left(Q_{Z}^{111}, Q_{X}^{111}\right)$ projections of the 3D $111 \mathrm{Bragg}$ reflection of NW4 that has been exposed for $3 \mathrm{~h}$. In contrast to NW2, the respective Bragg reflection stays constant in intensity and shows almost no variation along all reciprocal-space directions defined by $Q_{X}^{111}, Q_{Y}^{111}$ and $Q_{Z}^{111}$. Qualitatively, NW4 shows very minor tilting, no intensity decay and no lattice expansion.

In order to quantify the NW tilt, the RSMs in the $\left(Q_{Y}^{111}\right.$, $Q_{X}^{111}$ ) plane were integrated along $Q_{Y}^{111}$ and $Q_{X}^{111}$ one at a time and the resulting integrated line scans were fitted by multiGaussian functions. In Figs. $6(a)$ and $6(b)$, the angular tilts of the NW along $Q_{Y}^{111}$ and $Q_{X}^{111}$ are denoted by $\alpha_{y}$ and $\alpha_{x}$, respectively, tracing their variation as a function of exposure time and thus of the absorbed X-ray dose. Details on the calculation of the absorbed dose are given in the supporting information (Part 3). At an exposure time of $48 \mathrm{~min}$, it can be clearly seen that, for NW2, P and T tilt in opposite directions 


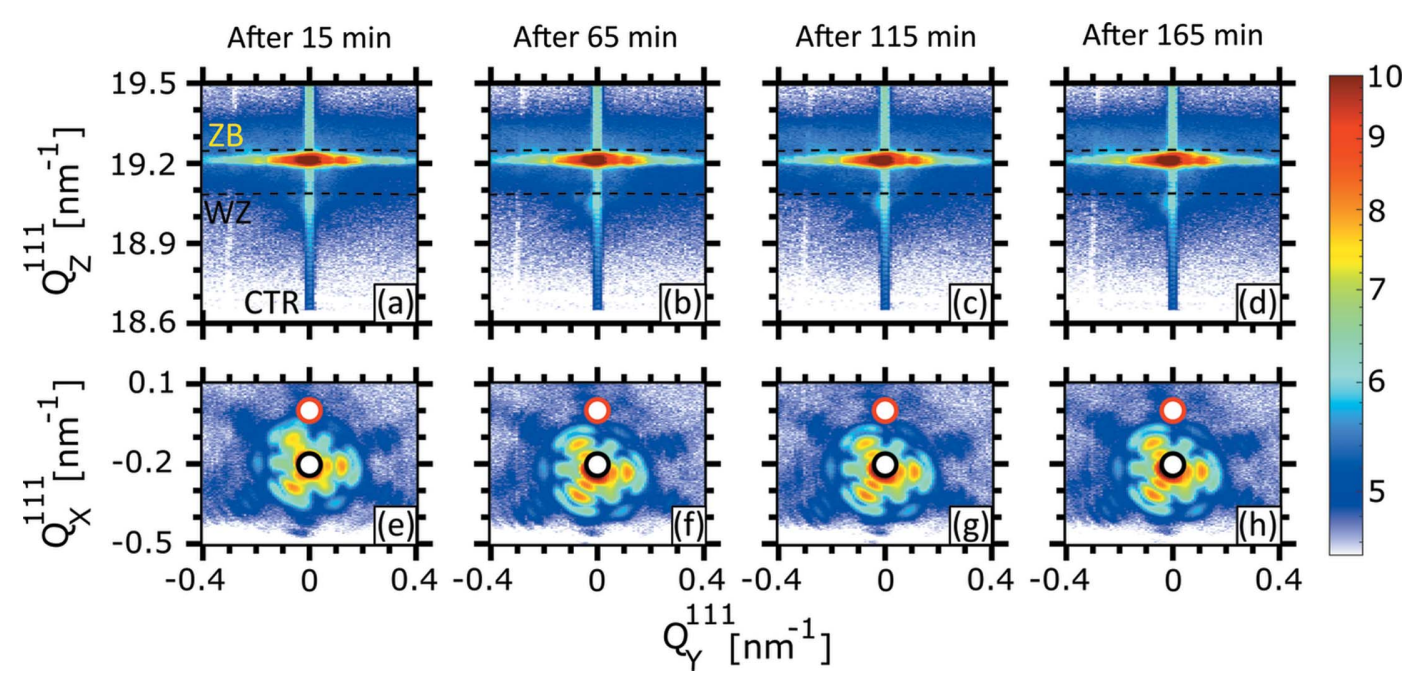

Figure 5

$(a)-(d) 2 \mathrm{D}$ projections of the 111 Bragg reflection of NW4, exposed under He atmosphere, in the $\left(Q_{Z}^{111}, Q_{Y}^{111}\right)$ reciprocal-space plane. The two dashed curves in $Q_{Z}^{111}$ represent the ZB (top) and WZ (bottom) Debye-Scherrer rings. $(e)-(h) 2 \mathrm{D}$ projections of the 111 Bragg reflection in the $\left(Q_{Y}^{111}, Q_{X}^{111}\right)$ reciprocal-space plane. The red and black circles represent the positions of the Si CTR and the initial NW tilt, respectively. The time at which each RSM acquisition was started is mentioned at the top.

indicating a small bending. The heavily illuminated part $\mathrm{P}$ tilts by $0.1^{\circ}$ in $\alpha_{y}$ and $0.4^{\circ}$ in $\alpha_{x}$ with respect to the initial position, whereas the less illuminated part T tilts by $-0.2^{\circ}$ in $\alpha_{y}$ and $\alpha_{x}$ by the end of exposure. The intensity decay of the $111 \mathrm{Bragg}$ reflection is plotted in Fig. 6(c) showing an exponential decay. This curve has been calculated by integrating the intensity distribution of the $3 \mathrm{D}$ Bragg reflection along all three reciprocal-space vectors, $Q_{Z}^{111}, Q_{Y}^{111}$ and $Q_{X}^{111}$, while excluding the $\mathrm{Si}$ CTR. Finally, from the variation of the Bragg reflections in $Q_{Z}^{111}$, we were able to calculate the variation in the axial $c$ lattice parameter, $\Delta c$, as shown in Fig. $6(d)$. The unstrained axial lattice parameter of ZB GaAs $(a=5.65325 \AA)$ was considered as a reference value $(\Delta c=0)$. The WZ polytype is formed in the upper part of the NW (see Fig. S5a in the supporting information) when the Ga droplet is consumed at the end of the core growth (Rieger et al., 2013; Lähnemann et al., 2019). The WZ Bragg reflection undergoes a rapid thermal lattice expansion before disappearing after $92 \mathrm{~min}$ of exposure. Considering the thermal expansion coefficient of bulk GaAs, $6.4 \times 10^{-6} \mathrm{~K}^{-1}$ (Straumanis \& Kim, 1965; Pierron et al., 1966), the measured peak shift is associated with sample heating by about $\Delta T \simeq 450 \mathrm{~K}$ (see Fig. S $5 b$ in the supporting information). Based on SEM images, the evolution of this peak can be caused by tilting away from the Bragg condition
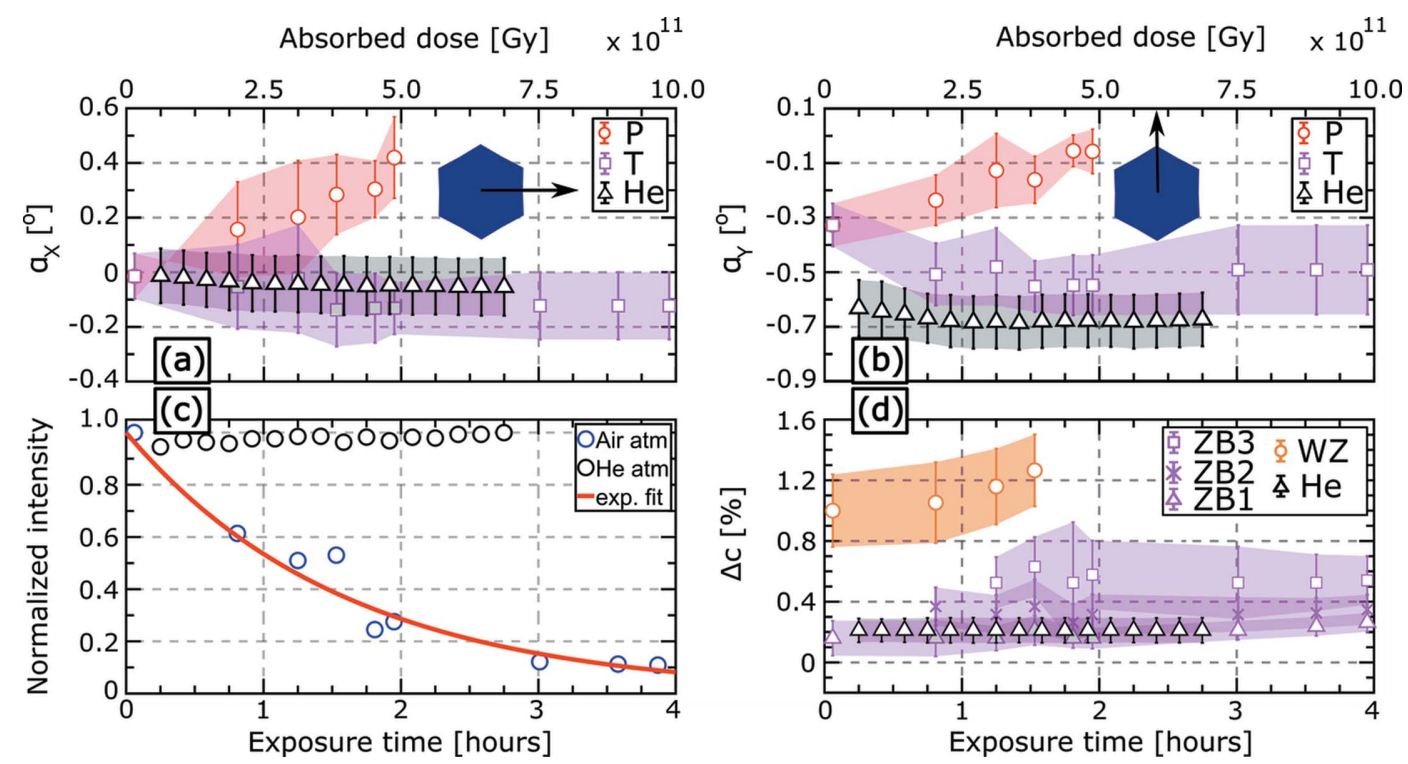

Figure 6

(a), (b) Tilt of NW2 (air) and NW4 (He) along $Q_{X}^{111}$ and $Q_{Y}^{111}$, respectively. The blue hexagon represents the NW cross section and the black arrow represents the tilt direction. Red data points correspond to section P, whereas the pink ones correspond to section T of NW2. The black points correspond to NW4, exposed for $3 \mathrm{~h}$ under He atmosphere. (c) Intensity decay of the integrated 111 Bragg reflection for the two NWs. The red curve is an exponential fit. Error bars are within the size of the dots. $(d)$ Variation in the $c$ lattice parameter. The orange points correspond to the WZ reflection. The three ZB sub-peaks, colored in pink, were named ZB1-ZB3 as indicated in Fig. 4(d). 
followed by a melting of this section. The main ZB Bragg reflection, visible at the beginning of exposure, splits into two sub-peaks after $48 \mathrm{~min}$ and then into three sub-peaks after $92 \mathrm{~min}$. Since the three sub-peaks originate from the main ZB reflection, they are referred to as $\mathrm{ZB} 1, \mathrm{ZB} 2$ and $\mathrm{ZB} 3$ in Fig. $6(d)$. After 92 min of exposure time, a thermal expansion gradient of about $0.5 \%$ is calculated comparing $\mathrm{ZB} 3$ and $\mathrm{ZB} 1$. As can be seen in Fig. 6(d), ZB3 undergoes the highest lattice expansion compared with the position of the original $\mathrm{ZB}$ reflection at the beginning of exposure. This reflects the impact of the peak and tail exposures of the primary beam on the $\mathrm{ZB}$ polytype as a function of its spatial position along the $\mathrm{NW}$ growth axis. Therefore, $\mathrm{ZB} 3$ is assumed to be the region located directly below the WZ segment and beneath it is ZB2 and then ZB1 as sketched in Fig. S5a in Part 4 of the supporting information. NW4, represented by black data points in Fig. 6, shows only negligible variation in $\alpha_{y}$ and $\alpha_{x}$, no decay in the integrated intensity of its measured 111 Bragg reflection and no axial lattice variation.

Overall, a total of $12 \mathrm{NWs}$ under air atmosphere and six NWs in He have been systematically exposed for different time intervals. The described results are representative for all the measured NWs. The speed at which the degradation in air proceeded varied between NWs, as differences in the alignment of the beam with respect to the NW lead to variations in the deposited dose rate. The diameter of all NWs exposed in air increased by $50-150 \mathrm{~nm}$ (30-100\% of initial diameter), while the CL was fully quenched after less than $2 \mathrm{~h}$. Besides NW2, melting was observed in one more NW exposed for $3 \mathrm{~h}$. For the NWs exposed under $\mathrm{He}$ atmosphere, barely any morphological changes or variation of the axial lattice parameters were consistently observed, while the degradation of the CL was significantly slowed down.

\section{Discussion}

Our experiments have revealed that significant radiation damage occurred for single $\mathrm{GaAs} /(\mathrm{In}, \mathrm{Ga}) \mathrm{As} / \mathrm{GaAs} \mathrm{NWs}$ exposed in the as-grown geometry to an $\mathrm{X}$-ray nanobeam with a photon flux of about $10^{10} \mathrm{~s}^{-1}$, provided by a third-generation synchrotron radiation facility under ambient conditions. The damage observed in air atmosphere, which is characterized by oxidation, morphological deformation and optical degradation, was significantly reduced when flushing the NWs by a continuous flow of He gas. Thus, the damage in air can be associated with the impact of ozone generated by the highly intense X-ray beam and the subsequent formation of oxide layers on the NW side planes (Flinn \& McIntyre, 1990; Lu et al., 1993; Hollinger et al., 1994). Lu et al. (1993) have concluded that ozone-induced oxidation of $\mathrm{GaAs}_{\mathrm{A}}(100)$ results in a stack of binary oxide layers, namely $\mathrm{Ga}_{2} \mathrm{O}_{3}, \mathrm{As}_{2} \mathrm{O}_{3}, \mathrm{As}_{2} \mathrm{O}_{5}$. In contrast, Hollinger et al. (1994) have shown that an amorphous, single-phase, non-stoichiometric ternary oxide is formed. This transformation of GaAs to an amorphous oxide leads to structural damage, which is in turn responsible for the loss in CL signal. Furthermore, it may inhibit the sufficient dissipation of the heat created by the X-ray beam into the surrounding atmosphere and result in a heating of the NWs.

The loss of CL emission from the embedded QW after $\mathrm{X}$-ray exposure is a direct consequence of the oxidation process. The CL intensity is highly sensitive to the presence of non-radiative defects. The interface of the growing oxide shell to the NW core is likely to induce strong non-radiative recombination (Küpers et al., 2019). However, the oxidation process may even introduce non-radiative centers deeper in the NW, for example by in-diffusion of O. Such defects could account for the locally reduced emission in the central segment of NW1 and the quenching of the luminescence in parts of NW4, where the morphological changes are more subtle. As the oxide shell grows at the cost of the outer GaAs barrier layer, the interface approaches the $\mathrm{QW}$, which will increase the loss of carriers. Even before the $30 \mathrm{~nm}$-thick shell is completely transformed and the oxidation of the QW starts, a complete loss of the CL signal can be expected. While the heating of the NW during the prolonged X-ray exposure could at some point lead to an interdiffusion of $\mathrm{Ga}$ and In atoms that degrades the previously well defined QW shell, the emission energy of such a wider (In,Ga)As shell with reduced In content would shift to higher energies, which is not observed experimentally. Therefore, we conclude that the oxidation is likely to be the main driving force for the quenching of the CL emission.

Concerning the melting of NW segments exposed for an extended period in air, the peak shifts in our nXRD experiments indicate a heating of up to $450 \mathrm{~K}$. Wallander \& Wallentin (2017) investigated the effect of X-ray-induced NW heating using time-resolved finite-element modeling. They studied the time-dependent response of single and repeated pulsed X-rays on an InP NW, either dispersed on a conductive $\mathrm{Si}_{3} \mathrm{~N}_{4}$ membrane or in the as-grown geometry. Thus, they could demonstrate that a thermal equilibrium in the NW during X-ray exposure is reached within tens of nanoseconds. The heat transport towards the substrate was identified as the main channel of heat dissipation for NWs dispersed horizontally on the substrate, as the temperature generated within the $\mathrm{NW}$ in the as-grown geometry was significantly larger. For the as-grown NWs, the authors concluded that heat transfer to the surrounding atmosphere through convection becomes the dominant cooling channel as the contact area to the substrate is small.

Additionally taking into account the oxide layer, this finding can also explain our results. The strong structural damage up to the melting of the top section observed under air atmosphere for NW2 clearly reflects that the temperature within NWs that have been exposed to X-rays under ambient conditions must be much higher compared with the NWs exposed in $\mathrm{He}$ atmosphere. While thermal equilibrium is reached on a nanosecond timescale, the structural damage occurs on the same slow timescale (hours) as the buildup of the oxide shell. It is thus reasonable to assume that the oxidation affects the heat dissipation and thereby leads to an increase in temperature. The formation of an oxide shell with lower heat conductivity would further reduce the contact area 
with the substrate. Even more importantly, the heat transfer to the surrounding atmosphere may be reduced by such a shell, which acts as a thermal resistor for heat transport.

Neglecting the continuous axial heat flow, the NW temperature created by the reduced heat transfer through the barrier can be estimated considering the $2 \mathrm{D}$ solution of the heat-transfer equation, namely the temperature gradient through a heat-resisting barrier. It is given by (Meschede, 2004)

$$
T_{\mathrm{NW}}=T_{0}+\frac{P d}{A \lambda}
$$

where $P$ is the heating power from the $\mathrm{X}$-ray beam, $A$ is the illuminated surface area (the cross section irradiated by the $\mathrm{X}$-ray beam), $\lambda$ is the heat conductivity and $d$ is the thickness of the barrier constituted by the oxide shell formed around the NW surface. The thermal conductivity coefficient of GaAs $\lambda^{\mathrm{GaAs}}$ is about $50 \mathrm{~W}(\mathrm{~m} \mathrm{~K})^{-1}$ (Maycock, 1967; Sze, 1981). $\lambda^{\beta-\mathrm{Ga} 2 \mathrm{O} 3}$ of crystalline $\mathrm{Ga}_{2} \mathrm{O}_{3}$ is found to range between 9.5 and $22.5 \mathrm{~W}(\mathrm{~m} \mathrm{~K})^{-1}$ depending on orientation (Guo et al., 2015; Handwerg et al., 2016), while for amorphous oxides and amorphous semiconductors $\lambda$ is expected to be at least one order of magnitude smaller (Yoshikawa et al., 2013; Wingert et al., 2016). Since $T_{\mathrm{NW}}$ is linear in $d$, but inversely proportional to $\lambda$, the increase in temperature can be explained by the increasing thermal resistance of the growing oxide layer. Based on equation (1), a numerical estimate provides a figure for $T_{\mathrm{NW}}-T_{0}$ on the order of $100 \mathrm{~K}$ for a conservative estimate of $\lambda$, but a higher value if a smaller value for $\lambda$ is assumed (see Part 4 of the supporting information). On the other hand, considering the massive electronic excitation by the X-ray beam and subsequent electron-electron and electron-phonon interaction where a major part of the energy is transferred to the lattice, non-thermal melting may additionally contribute to the increase of the lattice temperature. These estimates support our conclusion that the observed shift of the Bragg reflections is by various sources of heating for samples covered by an oxide layer. In contrast, NWs exposed under $\mathrm{He}$ atmosphere show a reduced oxide deposition and subsequently a higher heat transfer to the surrounding $\mathrm{He}$ atmosphere, which keeps the temperature of the NW at a lower level. In addition, the heat dissipation from the NW side facets to the gas atmosphere through convection is more effective under He compared with air because of the higher thermal conductivity of $\mathrm{He}\left[0.1513 \mathrm{~W}(\mathrm{~m} \mathrm{~K})^{-1}\right]$ compared with air $\left[0.024 \mathrm{~W}(\mathrm{~m} \mathrm{~K})^{-1}\right]$ (Yang et al., 2010).

\section{Related literature}

The following references are cited in the supporting information: Dhanaraj et al. (2010), Heyn \& Jesson (2015).

\section{Summary}

We have demonstrated that continuous illumination of compound semiconductor NWs under air atmosphere by an $\mathrm{X}$-ray beam focused to a sub-micrometre spot size at a fixed beam position can induce profound changes in the structure and morphology of the NWs with severe impact on the emission properties of embedded core-shell QWs as shown by CL measurements. As demonstrated, the exposure of groupIII arsenide NWs under ambient conditions for $1 \mathrm{~h}$ leads to a local degradation of the CL emission and to an about $30 \%$ increase of the NW diameter. Major structural changes, including tilting, lattice expansion and an intensity reduction of the Bragg reflection, already set in at this stage. NWs exposed for $2 \mathrm{~h}$ show no more CL emission. When the exposure time exceeds $3 \mathrm{~h}$, a melting of parts of the NWs is possible. The damage to the NWs can be largely mitigated by measurements under He atmosphere. For exposures up to $3 \mathrm{~h}$, neither a variation in the axial lattice spacing nor an intensity decay of the measured Bragg reflection are observed. The CL is quenched at the exposed area, but not necessarily along the whole length of the NW. Therefore, and with the help of EDX measurements, we attribute the structural changes to oxidation of GaAs under the influence of ozone created by the $\mathrm{X}$-ray beam under ambient conditions. This oxidation limits the heat transfer from the NW side facets to the surrounding atmosphere and leads to significant sample heating and thus lattice expansion and eventually a melting of parts of the NW. The reduced CL emission is attributed to non-radiative recombination at the interface between GaAs and amorphous oxides, probably combined with the introduction of nonradiative centers deeper in the NW.

The presented results will have a significant impact on experiments to be performed in future nanobeam stations at third- and fourth-generation synchrotron facilities. In general, it would be desirable to avoid beam damage by performing the experiment under inert gas atmosphere as shown in this report. This approach may be complemented by an improved contact between the NW and the substrate, a continuous sample cooling or an attenuation of the beam. With the emergence of artificial intelligence in scientific applications and analysis, raw 2D intensity detector frames of these diffraction patterns, with proper metadata context, could be used as training data for artificial intelligence recognition of damage onset using machine learning algorithms, forming a new scope of automation for future scattering experiments.

\section{Acknowledgements}

We acknowledge DESY (Hamburg, Germany), a member of the Helmholtz Association HGF, for the provision of experimental facilities. Parts of this research were carried out at the PETRA III beamline P08. Open access funding enabled and organized by Projekt DEAL.

\section{Funding information}

This work was supported by the DFG under grant Pi218/38.

\section{References}

Al Hassan, A., Davtyan, A., Küpers, H., Lewis, R. B., Bahrami, D., Bertram, F., Bussone, G., Richter, C., Geelhaar, L. \& Pietsch, U. (2018b). J. Appl. Cryst. 51, 1387-1395. 
Al Hassan, A., Lewis, R. B., Küpers, H., Lin, W.-H., Bahrami, D., Krause, T., Salomon, D., Tahraoui, A., Hanke, M., Geelhaar, L. \& Pietsch, U. (2018a). Phys. Rev. Mater. 2, 014604.

Biermanns, A., Rieger, T., Bussone, G., Pietsch, U., Grützmacher, D. \& Ion Lepsa, M. (2013). Appl. Phys. Lett. 102, 043109.

Boller, K., Haelbich, R.-P., Hogrefe, H., Jark, W. \& Kunz, C. (1983). Nucl. Instrum. Methods Phys. Res. 208, 273-279.

Claeys, C. \& Simoen, E. (2002). Radiation Effects in Advanced Semiconductor Materials and Devices, Vol. 57, pp. 245-280. Springer Series in Materials Science. Berlin, Heidelberg: SpringerVerlag.

Dhanaraj, G., Byrappa, K., Prasad, V. \& Dudley, M. (2010). Springer Handbook of Crystal Growth. Berlin, Heidelberg: Springer-Verlag.

Flinn, B. J. \& McIntyre, N. S. (1990). Surf. Interface Anal. 15, 19-26.

Guo, Z., Verma, A., Wu, X., Sun, F., Hickman, A., Masui, T., Kuramata, A., Higashiwaki, M., Jena, D. \& Luo, T. (2015). Appl. Phys. Lett. 106, 111909.

Handwerg, M., Mitdank, R., Galazka, Z. \& Fischer, S. F. (2016). Semicond. Sci. Technol. 31, 125006.

Heyn, Ch. \& Jesson, D. E. (2015). Appl. Phys. Lett. 107, 161601.

Hill, M. O., Calvo-Almazan, I., Allain, M., Holt, M. V., Ulvestad, A., Treu, J., Koblmüller, G., Huang, C., Huang, X., Yan, H., Nazaretski, E., Chu, Y. S., Stephenson, G. B., Chamard, V., Lauhon, L. J. \& Hruszkewycz, S. O. (2018). Nano Lett. 18, 811-819.

Hollinger, G., Skheyta-Kabbani, R. \& Gendry, M. (1994). Phys. Rev. $B, 49,11159-11167$.

Küpers, H., Corfdir, P., Lewis, R. B., Flissikowski, T., Tahraoui, A., Grahn, H. T., Brandt, O. \& Geelhaar, L. (2019). Phys. Status Solidi $R R L, \mathbf{1 3}, 1800527$.

Küpers, H., Lewis, R. B., Tahraoui, A., Matalla, M., Krüger, O., Bastiman, F., Riechert, H. \& Geelhaar, L. (2018). Nano Res. 11, $2885-2893$.

Lähnemann, J., Hill, M. O., Herranz, J., Marquardt, O., Gao, G., Al Hassan, A., Davtyan, A., Hruszkewycz, S. O., Holt, M. V., Huang, C., Calvo-Almazán, I., Jahn, U., Pietsch, U., Lauhon, L. J. \& Geelhaar, L. (2019). Nano Lett. 19, 4448-4457.

Leake, S. J., Chahine, G. A., Djazouli, H., Zhou, T., Richter, C., Hilhorst, J., Petit, L., Richard, M.-I., Morawe, C., Barrett, R., Zhang, L., Homs-Regojo, R. A., Favre-Nicolin, V., Boesecke, P. \& Schülli, T. U. (2019). J. Synchrotron Rad. 26, 571-584.

Lioliou, G. \& Barnett, A. M. (2016). Nucl. Instrum. Methods Phys. Res. A, 836, 37-45.

Lu, Z. H., Bryskiewicz, B., McCaffrey, J., Wasilewski, Z. \& Graham, M. J. (1993). J. Vac. Sci. Technol. B, 11, 2033-2037.
Martínez-Criado, G., Villanova, J., Tucoulou, R., Salomon, D., Suuronen, J.-P., Labouré, S., Guilloud, C., Valls, V., Barrett, R., Gagliardini, E., Dabin, Y., Baker, R., Bohic, S., Cohen, C. \& Morse, J. (2016). J. Synchrotron Rad. 23, 344-352.

Mastropietro, F., Eymery, J., Carbone, G., Baudot, S., Andrieu, F. \& Favre-Nicolin, V. (2013). Phys. Rev. Lett. 111, 215502.

Maycock, P. D. (1967). Solid-State Electron. 10, 161-168.

Meschede, D. (2004). Gerthsen Physik, 22nd ed. Berlin, Heidelberg: Springer-Verlag.

Peña, F. de la, et al. (2019). HyperSpy v1.5.2. DOI: 10.5281/ zenodo.592838.

Pierron, E. D., Parker, D. L. \& McNeely, J. B. (1966). Acta Cryst. 21, 290.

Pietsch, U., Holy, V. \& Baumbach, T. (2004). High-resolution X-ray Scattering: From Thin Films and Multilayers. Advanced Texts in Physics, 2nd ed. New York: Springer-Verlag.

Polvino, S. M., Murray, C. E., Kalenci, Ö., Noyan, I. C., Lai, B. \& Cai, Z. (2008). Appl. Phys. Lett. 92, 224105.

Rieger, T., Lepsa, M. I., Schäpers, T. \& Grützmacher, D. (2013). J. Cryst. Growth, 378, 506-510.

Seeck, O. H., Deiter, C., Pflaum, K., Bertam, F., Beerlink, A., Franz, H., Horbach, J., Schulte-Schrepping, H., Murphy, B. M., Greve, M. \& Magnussen, O. (2012). J. Synchrotron Rad. 19, 30-38.

Sellin, P. J. \& Vaitkus, J. (2006). Nucl. Instrum. Methods Phys. Res. A, 557, 479-489.

Shi, X., Xiong, G., Huang, X., Harder, R. \& Robinson, I. (2012). New J. Phys. 14, 063029.

Smolyanskiy, P., Bergmann, B., Chelkov, G., Kotov, S., Kruchonak, U., Kozhevnikov, D., Sierra, Y. M., Stekl, I. \& Zhemchugov, A. (2018). J. Instrum. 13, T02005.

Stankevič, T., Dzhigaev, D., Bi, Z., Rose, M., Shabalin, A., Reinhardt, J., Mikkelsen, A., Samuelson, L., Falkenberg, G., Vartanyants, I. A. \& Feidenhans'l, R. (2015). Proc. SPIE, 9592, 95920D.

Straumanis, M. E. \& Kim, C. D. (1965). Acta Cryst. 19, 256-259.

Sze, S. M. (1981). Physics of Semiconductor Devices, 2nd ed. New York: Wiley.

Wallander, H. \& Wallentin, J. (2017). J. Synchrotron Rad. 24, 925-933.

Wingert, M. C., Zheng, J., Kwon, S. \& Chen, R. (2016). Semicond. Sci. Technol. 31, 113003.

Yang, J., Tan, C. P. \& Ong, E. H. (2010). Proc. IMECE, 37622, 155159.

Yoshikawa, T., Yagi, T., Oka, N., Jia, J., Yamashita, Y., Hattori, K., Seino, Y., Taketoshi, N., Baba, T. \& Shigesato, Y. (2013). Appl. Phys. Express, 6, 021101. 